\title{
Archéopages
}

Archéopages

Archéologie et société

34 | 07/2012

Campagnes

\section{Le bocage. Un paysage rural à la lumière des études archéologiques et archéogéographiques}

\section{Magali Watteaux}

\section{(2) OpenEdition}

1 Journals

Édition électronique

URL : https://journals.openedition.org/archeopages/402

DOI : 10.4000/archeopages.402

ISSN : 2269-9872

Éditeur

INRAP - Institut national de recherches archéologiques préventives

Édition imprimée

Date de publication : 1 février 2012

Pagination : 64-72

ISSN : 1622-8545

\section{Référence électronique}

Magali Watteaux, «Le bocage. Un paysage rural à la lumière des études archéologiques et

archéogéographiques », Archéopages [En ligne], 34 | 07/2012, mis en ligne le 01 juillet 2012, consulté le

21 janvier 2022. URL : http://journals.openedition.org/archeopages/402 ; DOI : https://doi.org/

10.4000 /archeopages. 402 


\section{Le bocage}

\section{Un paysage rural à la lumière des études archéologiques et archéogéographiques}

Magali WatteaUX UmR 7041 «Archéologies et Sciences de l'Antiquité», CEAUCP, Université de Coimbra (Portugal)

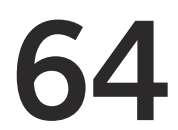

Depuis Marc Bloch le bocage est considéré comme l'un des paysages ruraux et agraires au fondement de la typologie paysagère de la France, avec l'openfield et les champs irréguliers du Midi. Il est défini par les géographes comme un paysage de haies végétales - souvent avec talus et fossé associé à un habitat dispersé, à un dense réseau de chemins, à un régime agraire considéré comme plutôt individualiste et à une forme relativement massive et irrégulière des parcelles, s'opposant ainsi, point par point, au paysage d'openfield [ill. 1]. Au Moyen Âge, le terme « bocage » avait le sens de pays boisé (Zadora-Rio, 1991).

\section{Le bocage, un objet d'étude pluridisciplinaire}

Tout au long du $\mathrm{Xx}^{\mathrm{e}}$ siècle, cet objet rural a suscité de nombreuses études tant chez les géographes que chez les historiens et les archéologues, mais aussi chez les agronomes, écologues, etc. Le binôme bocage/openfield est même devenu l'un des moteurs de la recherche sur les paysages ruraux en histoire et géographie. La question de sa genèse a en particulier provoqué des débats parmi les historiens et archéologues (Watteaux, 2005). Les érudits du XIX ${ }^{\mathrm{e}}$ siècle savaient déjà que le bocage n'avait pas toujours existé et les géographes ont toujours affirmé qu'il n'était pas originel, du moins pas pour toutes les régions. Puis les historiens ont proposé différentes hypothèses plutôt anciennes : âge du Fer, haut Moyen Âge ou Moyen Âge central dans le cadre des « grands défrichements ». Aujourd'hui, ils font apparaître le bocage au plus tôt à la fin du Moyen Âge et insistent sur le processus (progressif) de «l'embocagement » (Pichot, 2000 ; Antoine, 2002 ; Moriceau, 2003). Le bocage en tant que paysage agraire ne se met donc en place véritablement qu'à la période moderne, en relation avec les pratiques des élites laïques ou religieuses (Merle, 1958 ; Pichot, 200o) et le développement de l'élevage (Antoine, 2007, p. 194). Le maillage bocager présentait cependant, encore au début du XIX ${ }^{\mathrm{e}}$ siècle, un caractère inachevé et ce n'est qu'entre 1850 et 1950 qu'il atteint sa plus forte densité grâce à la mise en valeur des landes par chaulage et par l'intensification de l'élevage (ibid., p. 196-197).

Dans les régions de l'ouest de la France, le poids de cet objet est tel que toutes les études menées sur les paysages semblent devoir s'y rapporter. On peut s'en rendre compte dans un ouvrage non spécialiste de l'histoire, produit par des écologues du paysage qui y reprennent le récit linéaire élaboré par les géographes, analysant l'histoire du paysage dans ce secteur du territoire national selon un seul critère : le développement du bocage comme processus principal sur plusieurs millénaires [III. 2] (Burel et Baudry, 200o, p. XXVIII).

Et c'est cela qui finit par poser problème. Cet objet, à tout englober, finit par devenir trop «envahissant», c'est-à-dire trop systématique dans les discours. Ce «durcissement» de l'objet scientifique s'explique par sa valeur sociale actuelle. En effet, malgré les nuances apportées au modèle, le bocage est devenu un objet à caractère identitaire et occupe aujourd'hui une place de plus en plus affirmée dans les problématiques paléoenvironnementales en raison des débats qui tournent autour de sa conservation et de son évolution actuelle. Si cet enjeu contemporain est évidemment légitime, il ne peut pour autant continuer à recouvrir toute l'histoire passée des paysages de l'ouest. L'archéologie et les sciences paléoenvironnementales, par le nombre des données nouvelles qu'elles apportent, et l'archéogéographie, par le travail épistémologique de fond qu'elle a entrepris (Chouquer, 2007), permettent de dire 
1. La représentation traditionnelle de

l'openfield et du bocage.

En haut, plan cadastral

du finage du village

lorrain de Bleid. L'habitat

est groupé en un uvillage-

en-tas $n$ : les champs

se présentent en lanières,

nus, ouverts, groupés par

paquets en « quartiers 》

dont les noms sont

indiqués. On note la rareté

des chemins ruraux.

Quelques pièces de terre

sont situées près

du village, liées à une

propriété bourgeoise.

II n'y a pas de bois sur

le finage. En bas, calque

d'une photographie

aérienne IGN de la partie

est de la commune de

Languidie (Morbihan).
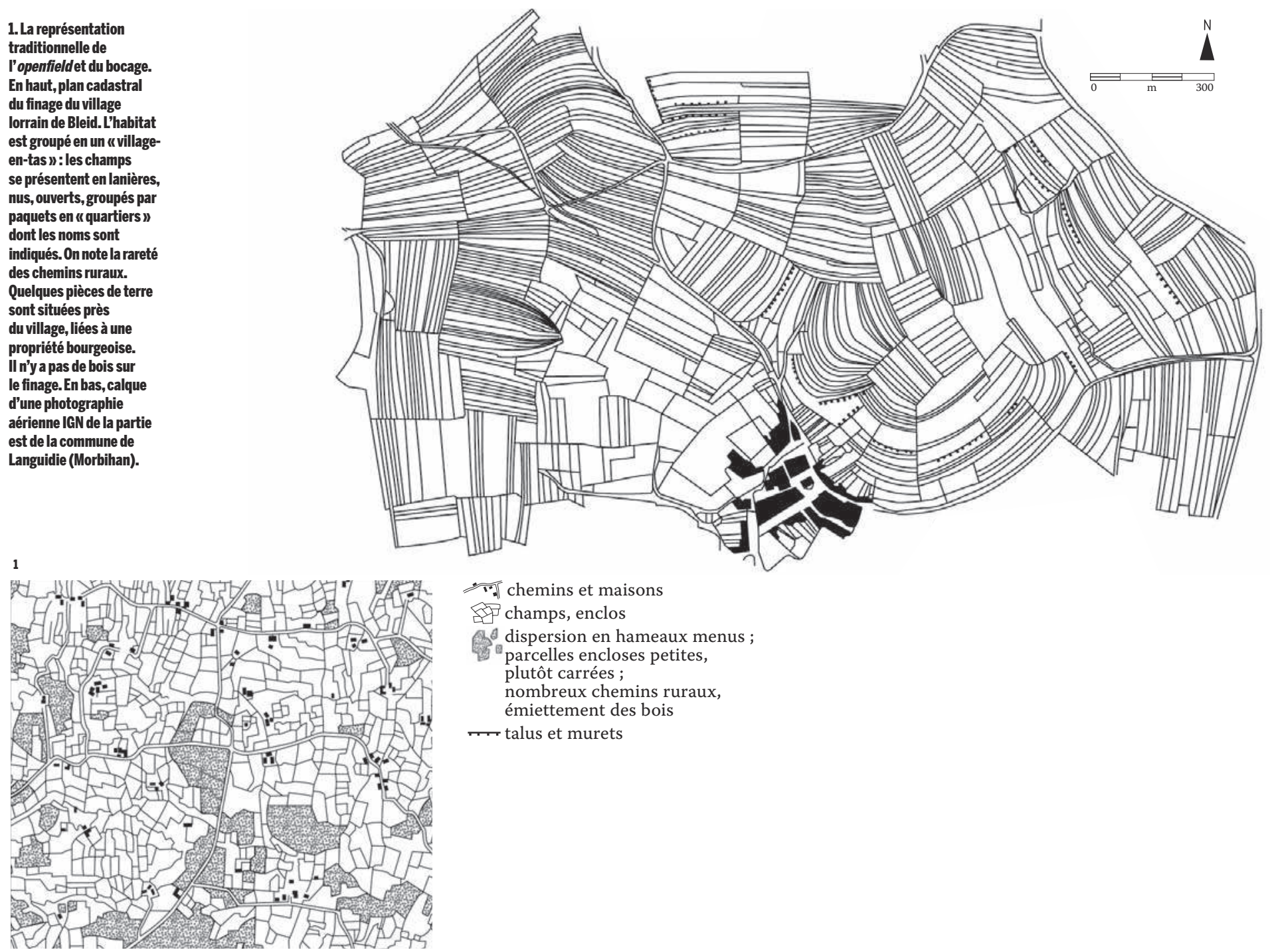

Themins et maisons

Shamps, enclos

dispersion en hameaux menus

parcelles encloses petites, plutôt carrées ;

.. talus et murets

2.a
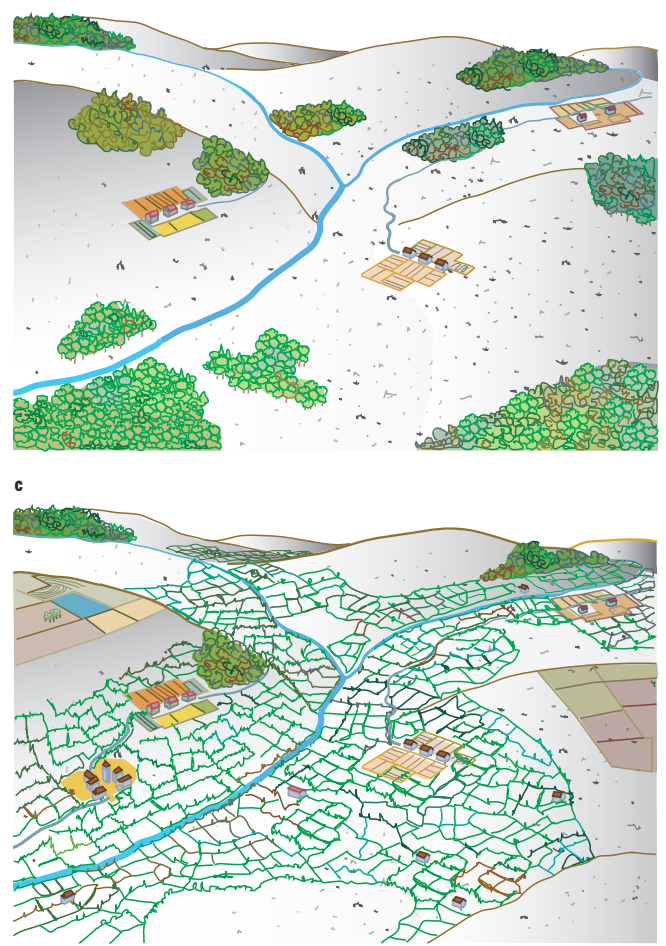

b
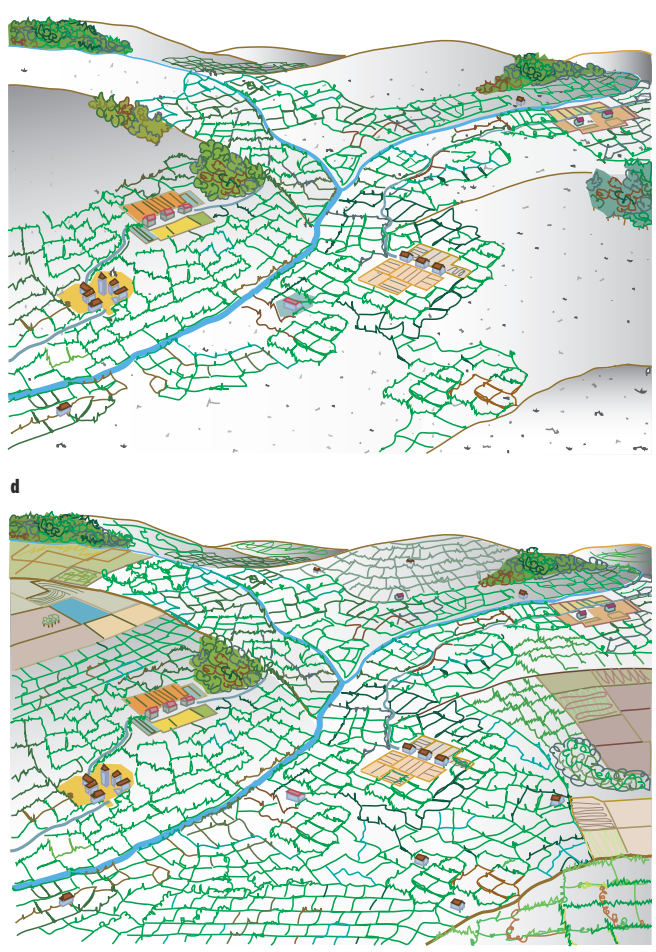

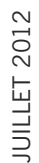

de la "Igenèse "

un bocage (d'après

P. Brunet, 1992, Atlas

des paysages ruraux

de France).

a. Le début du peuplement.

b. L'essor des fermes

familiales (x|'-x|l|' $\mathrm{S}$.)

c. La poussée des

métairies (Xve-xV|'s.)

d. L'achèvement du

bocage (xxe s.). 

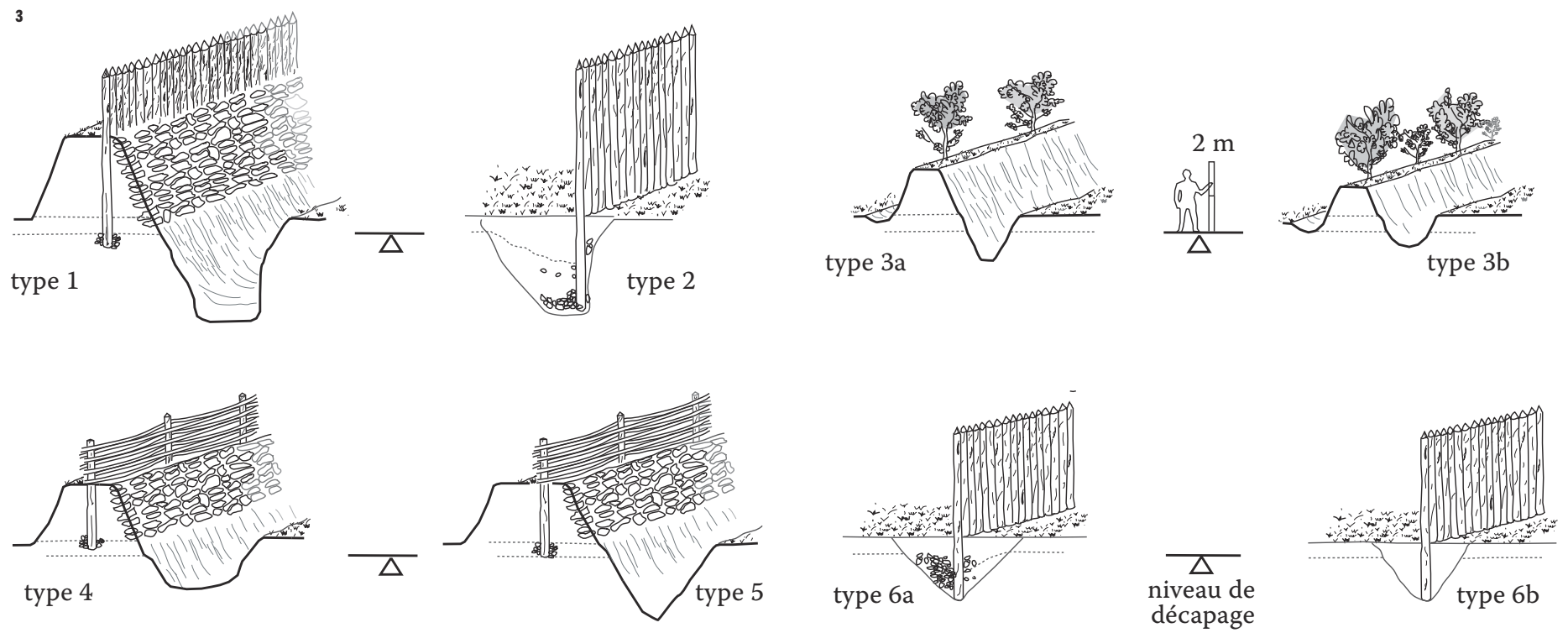

8
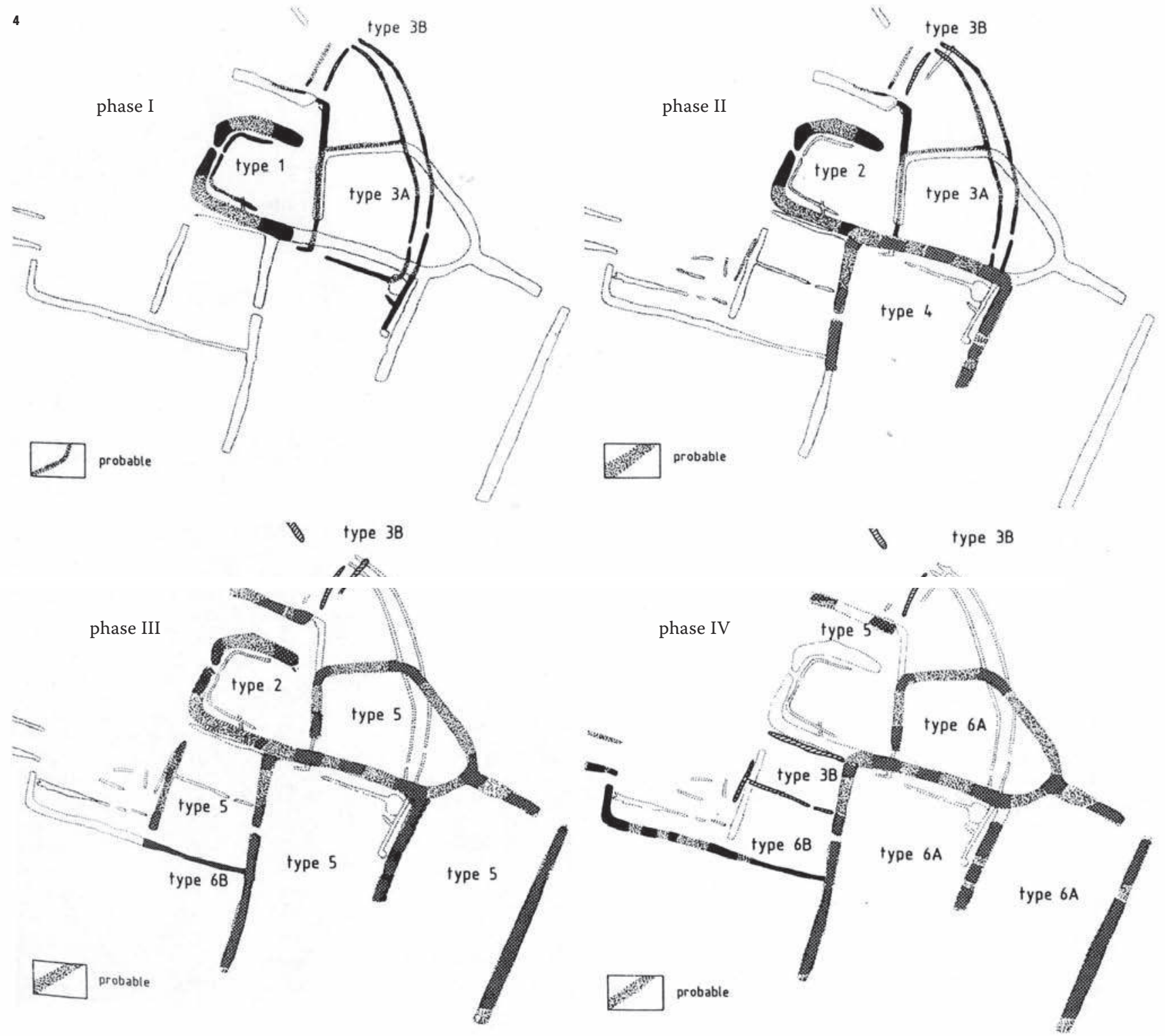
3. Les différents types de clôtures proposés par les archéologues pour la ferme du Boisanne. 4.Spatialisation en quatre phases et chronologie relative des différentes clôtures de la ferme du Boisanne quels sont les nouveaux objets qui émergent et remplacent en grande partie, avec profit, ce concept mal adapté de bocage pour l'étude des paysages dans la longue durée.

\section{Les acquis de l'archéologie préventive}

Sur le terrain, les archéologues retrouvent les traces des haies, des clôtures, des talus et des fossés parcellaires. Ils les organisent en ensembles et tentent d'en restituer la dynamique évolutive. L'apport de l'archéologie préventive a, dans ce domaine, été considérable, compte tenu de l'augmentation décisive des surfaces prospectées.

Arrêtons-nous à ce sujet sur l'exemple de la ferme gauloise du Boisanne à Plouër-sur-Rance (Côtesd'Armor), fouillée sous la direction d'Yves Menez (Menez, 1996). Les archéologues ont distingué les aménagements de ce modeste habitat rural du $\mathrm{VI}^{\mathrm{e}}$ siècle avant notre ère jusqu'au $\mathrm{III}^{\mathrm{e}}$ siècle de notre ère en six états successifs qui montrent que l'histoire de cet établissement est faite de nombreux réaménagements de clôtures (de types variés) ayant systématiquement respecté les mêmes axes [ill. 3 et 4]. Ainsi, à travers les changements substantiels du gisement dont l'archéologie retrace l'histoire, on assiste à une stabilisation progressive de la forme de l'occupation du site local. Les grandes lignes sont respectées. Les décalages chronologiques, visibles dans la chronique et la cartographie de ces transformations, n'empêchent donc pas, à un autre niveau, une transmission qui en limite les effets.

En d'autres lieux, les archéologues mettent en évidence des phases de création et des cas de transmissions parcellaires comme au Teilleul et à Louvaquint [ill. 5] sur la commune de Montours (Ille-et-Vilaine), fouillés sous la direction d'Isabelle Catteddu (Catteddu, 2001). La présence d'un gué et d'un chemin reliant les deux sites ainsi que la similitude d'orientation et d'organisation font supposer leur contemporanéité au moins partielle. Malgré l'abandon des habitats, une grande trame structure les deux gisements, de l'âge du Fer à nos jours, car elle est encore perceptible dans le parcellaire actuel. Les formes reportées sur le cadastre napoléonien - dont les fossés ont été retrouvés en fouille - présentent également la même orientation (isoclinie) et se trouvent souvent au même emplacement (isotopie) que les fossés anciens. Les données archéologiques illustrent donc ici les effets d'une transmission, dans la longue durée, d'une orientation morphologique entre un enclos gaulois, un parcellaire du haut Moyen Âge - qui se cale sur cette forme antérieure -, le parcellaire du XIX ${ }^{\mathrm{e}}$ siècle et les fossés actuels. La création parcellaire au haut Moyen Âge permet donc ici la transmission de l'état protohistorique jusqu'à l'état moderne. Évidemment les modelés, eux, évoluent. On parle, pour cela, de transmission dynamique (ou «transformission»). Ainsi, à Montours, d'un point de vue archéogéographique, le bocage «s'efface» derrière les dynamiques parcellaires. Le site de Châteaugiron (Ille-et-Vilaine), en cours d'étude par la même responsable d'opération ${ }^{\mathbf{1}}$ (Menez et Hinguant, 2010), amène aux mêmes constats, de manière encore plus spectaculaire et illustre à son tour la force des héritages dans la structuration de ces terroirs bocagers, avant l'âge du bocage agraire moderne.

\section{Les avancées des sciences paléoenvironnementales}

Les sciences paléoenvironnementales sont particulièrement intéressantes pour étudier le végétal. Récemment, la recherche en palynologie a franchi un seuil en mettant en évidence un signal pollinique propre aux haies, comme à Montours et à SaintGermain-en-Coglès (Marguerie et al., 2003, p. 119). Posée comme hypothèse en 2003 ( ibid.), la culture préférentielle du chêne et/ou du châtaignier dans le cadre d'un aménagement bocager est devenue aujourd'hui une position renforcée par les travaux de Dominique Marguerie et Jean-Charles Oillic (2007). Létude de Montours montre que ce pic de pollens spécifiques intervient à partir $d u X V^{e}$ siècle ce qui corrobore les connaissances des historiens sur la mise en place de ce paysage agraire. Le fait que la résolution temporelle des analyses soit très précise permet selon eux de privilégier cette hypothèse plutôt que celle d'un bref reboisement suivi d'une déforestation. Cependant, cette observation, réalisée à grande échelle et sur une seule colonne de prélèvement, ne permet pas, de leur propre avis, de passer à la reconstitution paysagère à plus petite échelle : «À ce stade de la pollinisation actuelle en milieu bocager, la mise en place d'un système bocager semble difficilement visualisable à travers une seule étude palynologique. Elle passe par la réalisation d'une étude spatiale selon plusieurs séquences polliniques organisées en coïncidence avec un supposé réseau fossile permettant de contrôler la variation du signal pollinique local. Si seule la colonne $\mathrm{C}_{2}$ avait été réalisée à Montours, il aurait été impossible de détecter l'existence de haies à chênes. » (Marguerie et Oillic, 2007, p. 117). D'autres études réalisées dans le nord-ouest ${ }^{2}$ vont dans le même sens (ibid., p. 117). Enfin, les données dendrochronologiques récentes semblent également confirmer ces observations en montrant une omniprésence des arbres émondés dans les constructions (Bernard et al., 2007, p. 219). Nous retrouverions donc, dans ces écofacts datés de la période moderne, la signature de ce paysage agraire qui se met progressivement en place à partir du Xv ${ }^{\mathrm{e}}$ siècle. Même si ces recherches restent à approfondir par d'autres analyses, elles augurent en tout cas d'ores et déjà de perspectives prometteuses pour l'identification de réseaux anciens de haies.

Il faut également mentionner une recherche qui fut un temps à la mode : l'analyse botanique des haies, lancée dans les années 1970 par le botaniste anglais Max Hooper (1976). Son idée était aussi simple que séduisante : la composition floristique des haies vives pourrait représenter un critère de datation de ces haies. Sa méthode consistait à effectuer un comptage de toutes les essences archéogéographique est en cours sur ce secteur. 2. Par D. Barbier (Inrap) A.-L. Cyprien et

A. Ouguerram.

\section{$\widehat{\sigma}$}




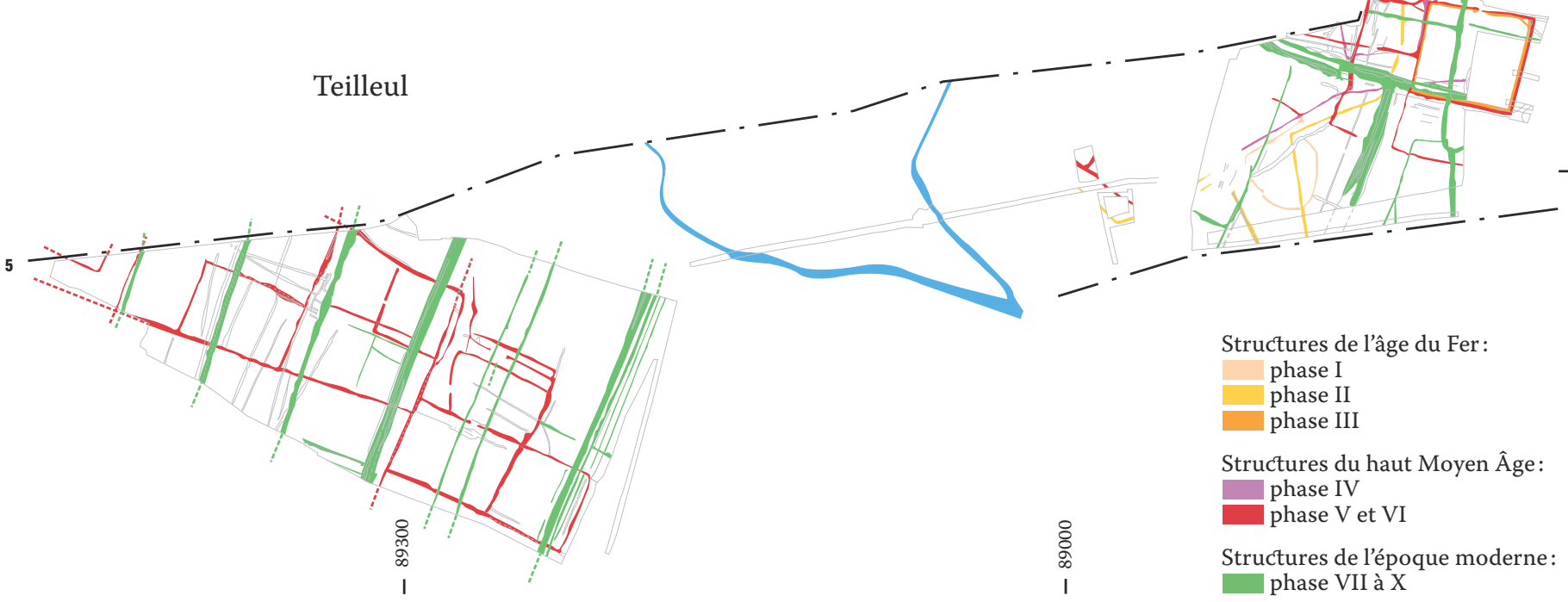

\begin{abstract}
5. Plan général des gisements

archéologiques du Teilleul et de Louvaquint (IIle-et-

Vilaine) avec localisation

des structures de l'âge du

Fer, du haut Moyen Âge et

de l'époque moderne.

6. Le grand réseau de

formation dominant la

plaine de Luçon-Fontenay.

7.Adaptation du réseau de

formation sud-vendéen

par distorsion aux

corridors hydro-végétalo-

parcellaires du nord-est
\end{abstract}

du secteur d'étude.
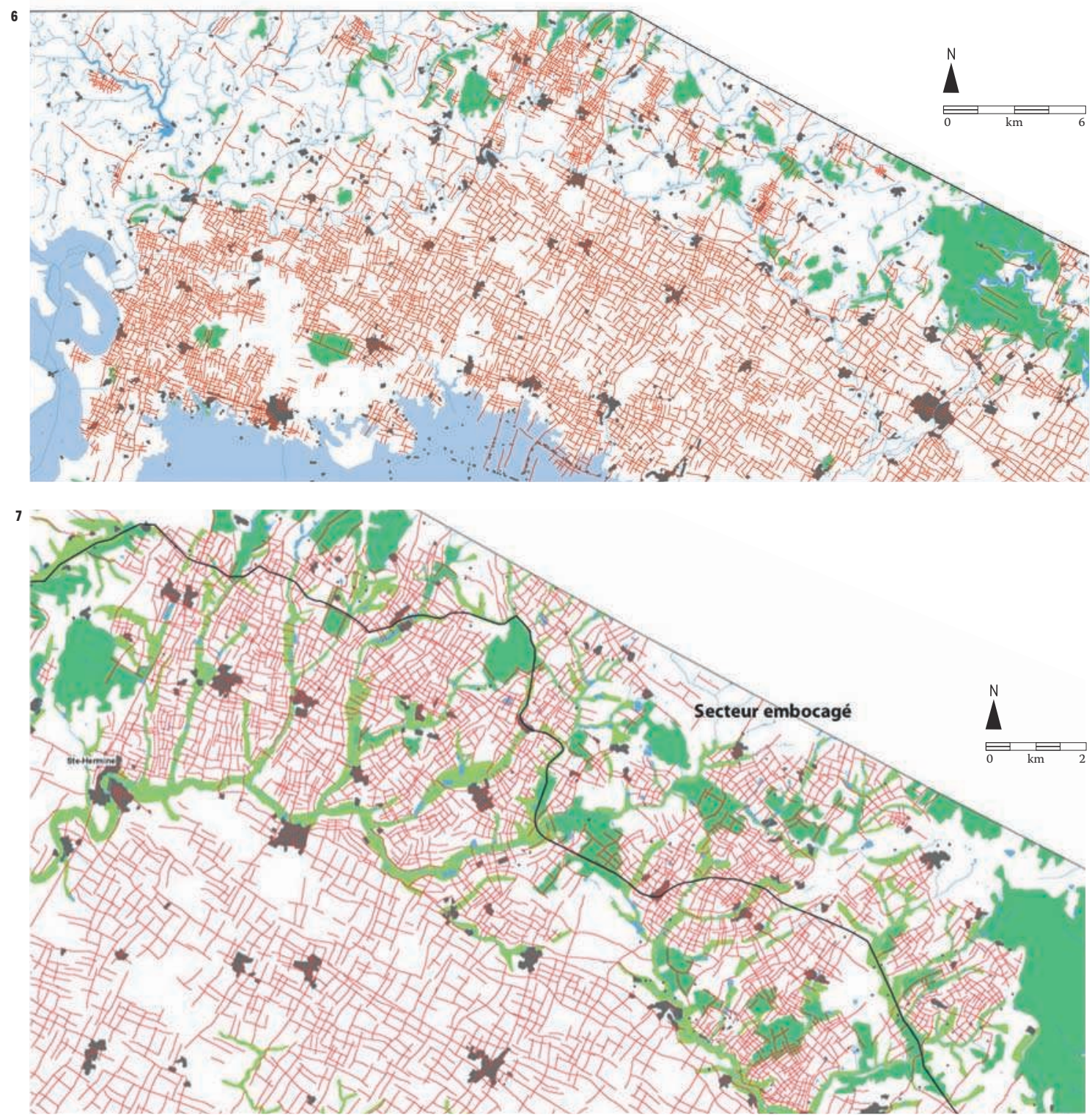
ligneuses par haie pour l'ensemble de l'aire étudiée. Elle reposait donc sur le présupposé qu'une haie est d'autant plus ancienne qu'elle renferme un nombre important d'espèces ligneuses (une nouvelle espèce en moyenne par siècle pour le sud de l'Angleterre). Par la suite, d'autres botanistes affinèrent la procédure et les paramètres à prendre en compte, en particulier la nature des espèces, les associations d'espèces et l'environnement proche de la haie. En France, dans les années 1980, des tentatives ont été menées pour appliquer cette méthode afin de dater les terroirs (Fourteau, 1983 ; Delelis-Dusollier, 1986 ; Perrein, 1987 et 1991). Christian Perrein a en particulier beaucoup travaillé dans cette optique, celle d'une « archéologie des bocages » ou «phytohistoire de la haie vive » (Perrein, 1991). Ces travaux, associés à des recherches de types historique, archéologique et archéogéographique, peuvent être intéressants mais il convient de rappeler qu'on ne peut confondre le modelé (haie végétale) et la forme en plan (limite parcellaire), et que l'étude d'une haie à grande échelle n'est pas celle d'un terroir ou territoire à plus petite échelle. En effet, dater une haie ne signifie pas dater son terroir ni le réseau bocager. La haie, en un sens, ne fait pas le bocage.

\section{Le paradoxe des approches archéologiques et paléoenvironnementales}

D'une manière générale, ces études archéologiques et paléoenvironnementales posent la question du lien entre des observations d'échelle locale et le bocage, au sens d'entité géographique et phénomène historique. Comment passe-t-on d'éléments issus de protocoles scientifiques très raffinés à un «métaobjet» historique? Ces analyses permettent de comprendre le fonctionnement des parcellaires fossoyés, des fonctions écologiques possibles des haies et du type de paysage végétal existant à telle ou telle période, voire - depuis récemment - de confirmer l'existence de nombreuses haies au sein de paysages cultivés, depuis la fin du Moyen Âge et surtout à l'époque moderne. Mais ces données permettent essentiellement d'atteindre une échelle spatiale locale qui n'est que très imparfaitement corrélée avec celle du paysage bocager (Burnouf et al., 2007, p. 125). Par ailleurs, les chercheurs décrivent plutôt des «ambiances » végétales que des paysages agraires au sens géographique. Le passage du diagramme pollinique (ou autre) au paysage se fait selon des modélisations qui, aussi évocatrices soient-elles, ne rendent pas compte du paysage planimétrique, c'est-à-dire de l'ensemble des formes parcellaires, routières et d'habitat qui caractérisent ce paysage.

Malgré la conscience des limites de leurs données pour parler d'espace planimétrique et malgré la mobilité chronologique des formations végétalo-parcellaires, les paléoenvironnementalistes ne remettent pas en question l'objet scientifique «bocage» en tant que cadre organisateur de leurs recherches. Au contraire, ils se moulent dans cette catégorie pourtant éloignée de leurs démonstrations pour parler du parcellaire et des milieux végétaux - mis à part les études portant sur la période moderne. C'est ce paradoxe qui explique qu'on trouve dans le programme du colloque Bocages et Sociétés (Antoine, Marguerie, 2007) des communications sur des périodes antérieures à la période moderne et sur des objets de nature bien différente que le bocage. Paradoxe que reconnaissent eux-mêmes les organisateurs : «La première [contribution] "La recherche des origines et le suivi des interactions société/paysage" - est probablement la plus étonnante puisqu'elle traite largement du bocage... avant le bocage !» (Antoine et al., 2007, p. 15). P.-R. Giot, M. Batt et M.-Th. Morzadec écrivaient pourtant déjà en $1982:$ : [...] les perspectives intéressantes de l'archéologie du paysage en Bretagne ne sont pas liées au bocage, mais bien à la recherche des données sur les paysages antiques et anciens, les zones de champs ouverts et les quelques talus anciens (ou talus maîtres).» (Giot et al., 1982, p. 5). «[Il faut] reconnaître que le plus gros du paysage bocager n'est, en définitive, pas aussi ancien qu'on se l'imaginait volontiers, c'est constater son intérêt plus secondaire pour l'archéologie du paysage. À ce point de vue, nous aurions peut-être mieux fait de dénommer notre opération "archéologie du paysage armoricain" sans faire allusion au bocage» (ibid., p. 29). Plus récemment, Patrick Naas (2007) est allé dans le même sens, rappelant que, dans les discussions au sein du groupe de recherche «Bocages armoricains et Sociétés », il est rapidement apparu que «les recherches sur les origines du bocage dans le nord-ouest de la France devaient être distinguées - au moins provisoirement - de la question des parcellaires anciens » (p. 66) et que «les recherches récentes en Bretagne semblent montrer que le bocage reste à ce stade une donnée peu pertinente pour l'étude des parcellaires antiques, même si les découvertes futures pourront partiellement rectifier ou nuancer ces propos » (p. 78). Dans ces conditions, on peut considérer que, si l'on continue d'intituler « bocage » l'objet des recherches, c'est par défaut, parce que les objets qui pourraient le remplacer se trouvent «sous» le bocage et qu'on ne les a pas identifiés comme tels. C'est justement l'un des objectifs de l'archéogéographie, qui postule la crise des objets de la géographie agraire d'antan et propose des recompositions scientifiques intégrant les récentes avancées de l'archéologie et de l'analyse morphologique des paysages.

\section{L'approche archéogéographique : le parcellaire}

Les nombreuses découvertes opérées grâce à l'archéologie préventive et leur analyse archéogéographique ont montré à quel point les formes des paysages se transmettent malgré les (et grâce aux) transformations depuis environ 3000 ans (Chouquer, 2000 et 2007 ; Robert, 2003). Même dans les régions bretonnes où l'on considérait que la règle était la rupture entre les établissements antiques et le bocage postérieur (assimilé au 


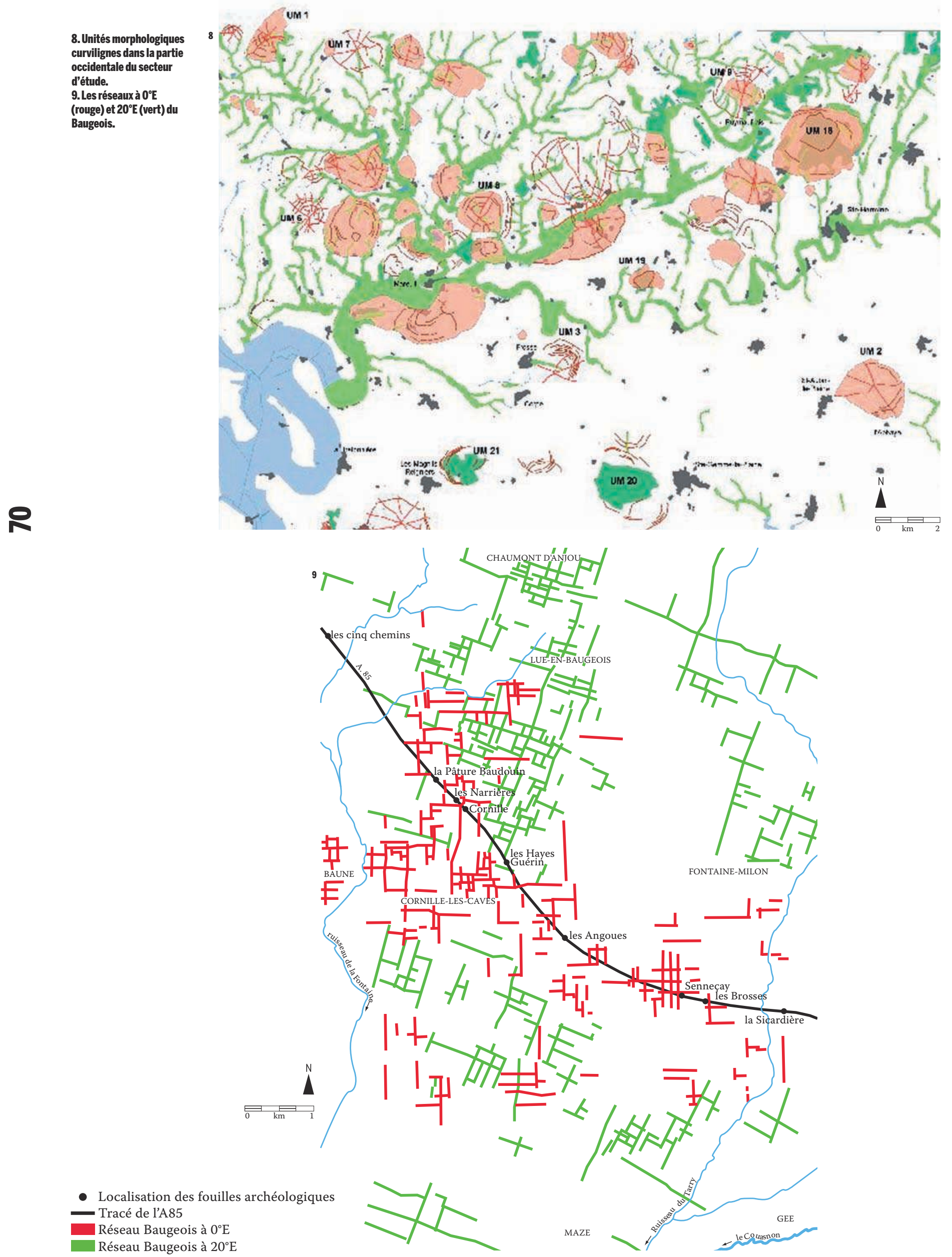


3. Consulter Watteaux,

2005, pour une

présentation de ce

dossier de Maurice

Gautier

4. Un quatrième, présent

sur le secteur, n'a pas fait

lobjet d'une étude

détaillée : le Marais

poitevin.

5. Dit « de formation»,

par opposition aux

réseaux planifiés.

6. Par l'expression « unité

morphologique

ponctuelle », on entend

le niveau intermédiaire

entre la masse parcellaire

tes grandes formes

organisatrices de la

planimétrie, comme

défini par Gérard

Chouker. Il s'agit d'un

mode de groupement des

parcelles en unités plus

vastes que les quartiers

de culture, mais d'un

périmètre circonscrit et

d'une superficie

relativement restreinte.

Les formes les plus

courantes sont les

ensembles circulaires, les

quadrillages

géométriques ponctuels,

les réseaux radiaux, les

limites curvilignes liées à

un fait topographique.

7. Plutôt qu'elles ne

renvoient directement

des systèmes agraires

particuliers. Samuel

Leturcq a bien montré

dans sa thèse que le

parcellaire est un

élément ni nécessaire ni

suffisant pour définir un

système agraire

d'openfield ou de bocage

(Leturcq, 2007) parcellaire du cadastre napoléonien, ce qui est d'ailleurs injustifié), des cas de transmission des orientations sont connus à plus petite échelle comme à Mauron-Yvel dans le Morbihan ${ }^{3}$ et à grande échelle (à Montours ou Châteaugiron). Cette lecture diachronique des formes parcellaires impose donc de distinguer la forme en plan et son modelé (ici, la haie). Cette dissociation n'est pas nouvelle : depuis la fin du XIX ${ }^{\mathrm{e}}$ siècle, les morphologues ont constaté la permanence des formes en plan, au-delà des transformations de leur modelé et de leur fonction (Robert, 2003). L'histoire de la formation du dessin parcellaire n'est donc pas réductible à celle du modelé bocager. Déjà André Meynier écrivait d'ailleurs : «Il [le bocage] meurt; mais le dessin cadastral ne change pas ; les champs gardent leur forme trapue, l'habitat reste dispersé » (Meynier, 1943, p. 163).

Récemment, l'analyse morphologique d'une fenêtre de $800 \mathrm{~km}^{2}$ dans le sud de la Vendée, enserrant une grande plaine et une zone embocagée a montré que la partition entre bocage et openfield, du point de vue de la planimétrie, n'était pas la plus pertinente (Watteaux, 2009 et sous presse). Trois espaces différents ${ }^{4}$ ont été identifiés et étudiés : - dans la plaine: un espace ouvert, très anciennement cultivé, dominé par un grand réseau de formes ${ }^{\mathbf{5}}$ [ill. 6], mais ajouré par des unités morphologiques ponctuelles ;

- entre Sainte-Hermine et la forêt de Mervent, zone en partie embocagée : le grand réseau de formation se prolonge ici au prix de distorsions qui permettent d'adapter ses limites au dense réseau des corridors « hydro-végétalo-parcellaires » [ill. 7], le tout associé à de nombreuses unités morphologiques ponctuelles ${ }^{6}$. Ici, les haies ne sont que l'habillage d'une partie de la trame générale organisant les formes du paysage.

- dans le nord-ouest du secteur, embocagé : la logique parcellaire est ici commandée par le réseau hydrographique, très varié dans ses orientations. Viennent s'y ajouter des dépendances locales aux chemins et l'impact des différents modes d'exploitation et de propriété des sols, métairies et borderies, qui créent des contrastes importants dans la planimétrie. Enfin, des unités morphologiques circonscrites [ill. 8], héritages d'aménagements de la période médiévale jusqu'au XIX ${ }^{\mathrm{e}}$ siècle, adoptent des formes curvilignes, radiales ou quadrillées. Des défrichements récents de landes $\left(\mathrm{XIX}^{\mathrm{e}} \mathrm{s}\right.$.) ajoutent une dernière touche au dessin d'ensemble. À aucun moment le modelé végétal de la haie permet de mieux comprendre cette diversité planimétrique.

Ces trois zones aux comportements morphologiques distincts témoignent de différents «styles parcellaires » ${ }^{7}$. Gérard Chouquer les définit comme l'« agencement du dessin parcellaire dont on pense qu'il indique des héritages morphologiques » (Chouquer, 200o, p. 190). Cette notion se rapproche de celle de «patron paysager » formulée par les écologues du paysage pour désigner l'arrangement spatial de la mosaïque (taches) et des réseaux (corridors), ce qui leur permet de comparer structuralement plusieurs paysages (Burel et Baudry, 200o, p. 70). Cette forte opposition du dessin parcellaire rend compte d'états antérieurs, d'héritages complexes. Cependant, les situations anciennes qui provoquent les héritages ne sont peut-être pas elles-mêmes les toutes premières. Elles sont seulement celles qui nous sont accessibles grâce aux documents. On peut, pour cette raison, dire que le secteur étudié comporte trois styles parcellaires hérités qui se distinguent grâce à l'analyse morphologique. Cette notion de «style parcellaire hérité» s'avère in fine plutôt féconde car elle permet de transcender la distinction traditionnelle entre bocage et openfield qui menait à une impasse, en proposant de réorganiser les données selon un autre découpage scientifique dont la logique est avant tout morphologique. Le parti pris initial de ne pas se concentrer sur un seul type de paysage - bocage ou plaine - s'est donc avéré payant car l'analyse morphologique a montré que cette distinction nétait pas pleinement opératoire. Il existe certes des différences très générales pour le réseau d'habitat (groupé/dispersé), pour les chemins (plus ou moins fragmentés), pour les masses parcellaires (très découpées et régulières/grands blocs irréguliers) et pour les modelés (ouverts/haies), mais il existe des degrés entre ces deux extrêmes qui viennent sérieusement nuancer le schéma :

— l'habitat peut être plus ou moins dispersé : de la ferme au petit bourg, en passant par le gros hameau ; - le réseau routier est moins atomisé qu'on le dit et intègre des itinéraires régionaux et supra-régionaux ; - il existe dans le bocage des ensembles parcellaires très découpés (borderies);

- la haie n'est pas inexistante dans la plaine et le secteur appelé, non sans raison « Entre-Plaine-etBocage » présente un paysage intermédiaire entre plaine ouverte et bocage ;

- des aménagements géométriques réguliers d'ampleur locale existent aussi dans le bocage ; - le grand réseau de formation est également organisateur de certaines zones embocagées. Concernant ce grand réseau de formes, la même chose a été observée en Anjou. Là, Gérard Chouquer et Anne Dodd-Opritesco ont mis en évidence, dans le cadre des opérations archéologiques préventives sur l'A85, deux vastes réseaux parcellaires de formation, à environ $20^{\circ} \mathrm{E}$ et $\mathrm{O}^{\circ} \mathrm{E}$, dans la région baugeoise (Maine-et-Loire) [ill. 9]. Ils présentent une orientation et des formes souples, et recoupent une dizaine de communes avec une densité qui tend à s'amoindrir vers le nord où se situent les coteaux boisés (Chouquer et al., 1997, p. 74). Ici aussi, rien ne permet d'identifier un «bocage» dans ces trames parcellaires auto-organisées, si ce n'est un modelé observé sur les cartes modernes et les images aériennes contemporaines.

Il ne s'agit pas de dire que cette lecture archéogéographique exclut celle fondée sur la dichotomie bocage/openfield - réalité moderne 
et actuelle - mais, au regard de la logique planimétrique, cette dernière n'est pas la plus pertinente. En Vendée, l'organisation des formes agraires transcende cette opposition des modelés. Le grand réseau de formation concerne en effet aussi bien la plaine, « l'Entre-Plaine-et-Bocage » que le bocage de l'est du secteur. Au contraire, le nordouest de la fenêtre répond à d'autres logiques. Cette région vendéenne, comme celle du Baugeois ou autour de Châteaugiron se rapproche donc du modèle qui émerge ailleurs, essentiellement en terres de champs ouverts : celui d'une forte création parcellaire antique au sens large (protohistoire et Antiquité romaine), création suivie d'une longue phase de résilience des formes, avec une transformation et une transmission variables et des changements de modelés dont le dernier en date est l'intense embocagement qui marque surtout l'époque moderne et contemporaine. L'histoire planimétrique de certaines régions bocagères ne se distingue ainsi plus radicalement des terroirs de champs ouverts dès lors que la forme parcellaire est isolée du modelé.

Dans le « bocage » des chercheurs se cachent en fait trois objets différents. Le premier est une formation hybride faite d'une part d'habitats, de parcellaire, de modelé (talus, fossés) et de végétal, d'autre part d'apparition tardo-médiévale et moderne, qualifiée de « bocage » par les géographes et transformée en type agraire historique médiéval et moderne par Marc Bloch et Roger Dion. C'est ce type que les médiévistes ont retenu dans leur exploitation de la dualité fondamentale des paysages agraires du Nord de la France qui se mettrait en place à partir du bas Moyen Âge au plus tôt. Le second objet est une représentation spéculaire de l'histoire du paysage et des formes dans la longue durée, qui consiste à qualifier uniformément de «bocage» tout le paysage passé, pour des raisons identitaires, sociales et historiques complexes. C'est, par exemple, le bocage aux racines celtiques des ethnologues et archéologues de jadis. C'est aussi ce qui explique qu'une recherche contemporaine sur le parcellaire ou le paysage doive encore passer par l'intitulé «bocage » pour être recevable. Enfin, et ce sont les véritables objets historiques à faire surgir, un ensemble de formes (le parcellaire) et d'habillages (modelé: talus, fossé, végétal, etc.) dont les dynamiques ne sont pas linéaires ni rigoureusement parallèles. Il y a un objet viaire et parcellaire, un objet-habitat, un objet-modelé bocager. Chacun a sa dynamique, avec des interactions, qui ne se résume pas à une histoire rigoureusement synchrone et identique. Le modelé bocager est un processus historique instable dans la durée, avec des avancées et des reculs (depuis la protohistoire), des phases fortes et des rémissions, qui fait que l'embocagement pourrait être ancien mais instable et non spécifique de périodes ou régions particulières. Comme le rappelle Jacques Baudry, les haies «sont communes à de nombreux paysages de la Terre» et sont très diversifiées dans leurs structures, leurs origines, leur répartition et leurs fonctions (Baudry et Perichon, 2007). Mais leur présence n'induit pas automatiquement l'existence d'un bocage. C'est pourquoi on peut tout à fait reconnaître l'existence de haies dès la préhistoire ou la protohistoire sans pour autant prétendre dater les douteuses origines d'un bocage. De même, l'identification de la pratique de l'émondage sur des arbres ne signifie pas nécessairement que nous sommes en présence d'arbres de haies bocagères ; elle a été mise en évidence au sein de woodlands en Angleterre ou encore dans des forêts de Galice ou de Suède (Bernard et al., 2007, p. 213). Enfin, l'historien moderniste Jean-Marc Moriceau rappelle, quant à lui, « qu'il n'y a pas de permanence rigide des structures du paysage dans le très long terme. Dans ses formes variées, le bocage a été le lieu où se sont développés des systèmes agraires assez différents» (Moriceau, 2003, p. 20). C'est pourquoi il faut distinguer le modelé paysager à base de haies, en tant que processus sur le long terme, de l'état identitaire qu'il a pris à partir de l'époque moderne et auquel il serait préférable de réserver le terme de «bocage». La question est de savoir pourquoi et comment des formes et des modelés deviennent un jour un «bocage» et quelle réalité recouvre ce terme. C'est cette forme «lourde» qui a conduit à une surdétermination de l'objet et donc à une illusion rétrospective. C'est en effet le saut de la représentation qui est important : l'invention d'une représentation bocagère surdéterminée a créé une réalité scientifique hypertrophiée, parce qu'elle vaut pour toute l'histoire du paysage de l'Ouest de la France. Mais les choses changent grâce aux travaux des uns et des autres. Notons en ce sens que, récemment, le médiéviste Daniel Pichot, révisant ses propres positions, a justement écrit : «Il serait aussi dangereux de penser le paysage des $\mathrm{XI}^{\mathrm{e}}$-XIII ${ }^{\mathrm{e}}$ siècles comme un pré-bocage dans une vision quasi téléologique» (Pichot, 2007, p. 263).

Ce constat montre que l'idée d'une réévaluation du type agraire bocager en tant que paysage central dans l'Ouest de la France, toutes périodes confondues, s'impose aujourd'hui. Dans le fond, chacun, dans le détail, convient de la rigidité ou même de la vacuité de ce type agraire. Reste à changer l'ensemble du dispositif pour recomposer les directions de recherche et faire véritablement émerger les nouveaux objets que les récentes données, archéologiques et archéogéographiques, laissent entrevoir. 


\section{Références bibliographiques}

Antoine A., 2002, Le paysage de l'historien. Archéologie des espaces bocagers de l'Ouest de la France à l'époque moderne, Rennes, Presses Universitaires de Rennes.

Antolne A., 2007, « D'un espace ouvert à un eśpace poreux. Bocage et élevage dans la France de l'Ouest du Moyen Âge au début du XIX ${ }^{\mathrm{e}}$ siècle ", in Antoine A., Marguerie D. (dir.), p. 185-200.

Antoine A. Marguerie D., Marchand J.-P., BAUdRY J., BurEL F., 2007, « Trente ans après... », in Antoine A., Marguerie M. (dir.), p. 9-21.

Antoine A., Marguerie D. (dir.), 2007, Bocages et Sociétés, Actes de colloque de Rennes, 23-30 septembre- $1^{\text {er }}$ octobre 2004, Rennes, Presses Universitaires de Rennes.

BAUdRY J., PÉRICHON S., 2007, « Les haies et les bocages dans le Monde : éléments de comparaison ", in Antoine A., Marguerie M. (dir.), p. 23-32.

Bernard V., Épaud F., Le Digol Y., 2007, «Bois de haies, bois de bocage, bois d'architecture », in ANTOINE A., MARguerie M. (dir.), p. 213-230.

Burel F., Baudry J., 200o, Écologie du paysage. Concepts, méthodes et applications, Paris-Londres-New York, Tec \& Doc.

Burnouf J., Ayala, G., Bernard, V., Bridault, A., Cammas, C., Castanet, C., Chouquer, G., Drucker, D., Fouillet, N., Jorda, C., Le Digol, Y., WATTEZ, J., 2007, « Des milieux et des hommes : méthodes d'étude en archéologies environnementales», in Demoule J.-P. (dir.), Larchéologie préventive dans le monde. Apports de l'archéologie préventive à la connaissance du passé, Actes du colloque de Paris, 30 septembre-1 ${ }^{\text {er }}$ octobre 2005, Paris, La Découverte, p. $117-130$.

CATTEDDU I. (dir.), 2001, Les habitats carolingiens de Montours et La Chapelle-Saint-Aubert (Ille-etVilaine), DAF, $\mathrm{N}^{\circ} 89$, Paris, Éd. de la MSH.

Chouguer G. (dir.), Carcaud N., David W., DoddOpritesco A., Mercier F., Pont C., Zadora-RioÉ. 1997, Autoroute A 85. Secctions de Corzé à Restigné. Étude des formes du paysage, Document Final de Synthèse, Tours, CNRS.

Chouquer G., 200o, Létude des paysages : essai sur leur forme et leur histoire, Paris, Errance.

CHOUQuer G., 2007, Quels scénarios pour l'histoire du paysage? Orientations de recherche pour l'archéogéographie, Coimbra/Porto, CEAUCP.
Delelis-Dusollier A., 1986, « Histoire du paysage par l'analyse de la végétation : l'exemple des haies », in Du pollen au cadastre, Actes du colloque de Lille, 10-12 octobre 1985, Hommes et Terres du Nord, 2/3, p. 110-115.

FourTEAU A.-M., 1983, « Botanique et paysage. Les haies d'une commune du Berry (Sagonne) », Archéologie médiévale, XIII, p. 155-183.

Giot P.-R., BatT M., Morzadec TH., 1982, Archéologie du paysage agraire armoricain, Rennes, Travaux $\mathrm{N}^{\circ} 40$, Laboratoire d'Anthropologie-Préhistoire-Protohistoire Quaternaire armoricains, Université de Rennes.

Hooper M., 1976, «Études historiques et biologiques des haies anglaises », in Les Bocages. Histoire, écologie, économie, Rennes, CNRS Éditions/ENSA Université de Rennes, p. 225-228.

LAVIGNE C., 2003, « De nouveaux objets d'histoire agraire pour en finir avec le bocage et l'openfield ", Études rurales, 167-168, p. 133-185.

LEBEAU R., 200o [1969], Les grands types de structure agraire dans le monde, Paris, Armand Colin.

LETURCQ S., 2007, Un village, la terre et ses hommes, Toury-en-Beauce (XII ${ }^{e}-\mathrm{XVII}{ }^{e}$ siècle), Paris, CTHS

Marguerie D., Antoine A., Thenail C., Baudry J., Bernard V., Burel F., Catteddu I., Daire M.-Y., Gautier M., Gebhardt A., Guibal F., Kergreis S., Lanos Ph., Le Ceeur D., Le DU L., Mérot P., NaAs P, Ouin A., Pichot D., Visset L., 2003, «Bocages armoricains et sociétés, genèse, évolution et interactions», in MuXart T., Vivien F.-D., Villalba B., Burnouf J. (dir.), Des milieux et des hommes: fragments d'histoires croisées, Paris, Elsevier, p. 115-131.

Marguerie D., Oillic J.-CH., 2007, « Pollens et haies du bocage dans le Nord-Ouest de la France », in Antoine A., Marguerie M. (dir.), p. 105-119.

Menez Y., Hinguant S., 2010, Fouilles et découvertes en Bretagne, Rennes, Éditions Ouest France/Inrap.

Menez Y., 1996, Une ferme de l'Armorique gauloise. Le Boisanne à Plouër-sur-Rance (Côtes-d'Armor) DAF, $\mathrm{N}^{\circ} 58$, Paris, éd. de la MSH.

MERLE L., 1958, La métairie et l'évolution agraire de la Gâtine poitevine de la fin du Moyen Âge à la Révolution, Paris, SEVPEN.

Meynier A., 1943, "Champs et chemins en Bretagne », in Conférences universitaires de Bretagne, organisées par la Faculté des Lettres de Rennes (1942-1943), Paris, Les Belles Lettres, p. 159-178.
MoriceAu J.-M., 2003, «Pérennité et fluctuations des paysages ruraux du XVII ${ }^{\mathrm{e}}$ au XIX ${ }^{\mathrm{e}}$ siècle : un regard d'ensemble», in Hervieu J.-P., DÉSIRÉ DIt GosSET G. BARRÉ É. (dir.), Les paysages ruraux en Normandie, Actes du colloque de Pont-Audemer, 17-20 octobre 2002, Annales de Normandie, Caen, p. 9-14.

NAAS P. 2007, « Aménagements agraires et parcellaires gallo-romains en Armorique », in Antoine A., Marguerie M. (dir.), p. 63-80.

Perrein CH., 1987, Contribution à l'archéologie des bocages: recherches méthodologiques sur l'utilisation des données botaniques de la haie vive, mémoire pour le diplôme de l'EHESS, Paris.

Perrein Ch., 1991, «Archéologie des bocages : phytohistoire de la haie vive », in GUILAINE J. (dir.), Pour une archéologie agraire. À la croisée des sciences de l'Homme et de la nature, Paris, Armand Colin, p. 223-257.

Piснот D., 2000, « Images du paysage : les bords de la Vilaine au Xvi siècle ", Mémoires de la Société d'histoire et d'archéologie de Bretagne, LXXVIII, p. 261-283.

Pichoт D., 2007, « Paysage et société féodale dans l'Ouest de la France », in Antoine A., MARguerie M.(dir.), p. $263-275$.

ROBERT S., 2003, « Comment les formes du passé se transmettent-elles? ?, Études rurales, 167-168, p. 115-132.

WATTEAUX M., 2005, « Sous le bocage, le parcellaire...», Études rurales, 175-176, p. 53-80.

WATTEAUX M. 2009, La dynamique de la planimétrie et des réseaux routiers en Vendée méridionale. Études historiographiques et recherches archéogéographiques, thèse de doctorat, Université Paris I, 3 vol. Téléchargeable sur : http://tel.archives-ouvertes.fr/ tel-oo421955/fr/.

WATTEAUX M., sous presse, « Lecture archéogéographique d'un bocage vendéen. Des héritages planimétriques variés, de l'âge du Fer au $\mathrm{xx}^{\mathrm{e}}$ siècle », Annales de Bretagne et des pays de l'Ouest, 118-4.

ZADORA-Rio É., 1991, « Les terroirs médiévaux dans le Nord et le Nord-Ouest de l'Europe », in Guilaine J. (dir.), Pour une archéologie agraire. À la croisée des sciences de l'Homme et de la nature, Paris, Armand Colin, p. 165-191. 